\title{
Proteomic analysis of aqueous humor from patients with branch retinal vein occlusion-induced macular edema
}

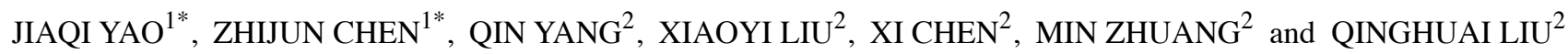 \\ ${ }^{1}$ Department of Ophthalmology, Nanjing Children's Hospital Affiliated to Nanjing Medical University; \\ ${ }^{2}$ Department of Ophthalmology, First Affiliated Hospital of Nanjing Medical University, Nanjing, Jiangsu 210029, P.R. China
}

Received June 9, 2013; Accepted September 17, 2013

DOI: $10.3892 /$ ijmm.2013.1509

\begin{abstract}
The mechanisms responsible for macular edema with branch retinal vein occlusion (BRVO) remain to be elucidated. It is known that the expression profile of certain proteins in the aqueous humor (AH) changes in some diseases. Accordingly, determining the expression of these $\mathrm{AH}$ proteins may aid in the understanding of their potential role in this pathogenesis. The aim of this study was to identify the possible mechanisms involved in the development of BRVO-induced macular edema. A proteomic analysis of the AH composition in the eyes of patients with BRVO was performed and compared with that in the eyes of patients with cataract (non-BRVO; controls). AH from 6 patients with macular edema due to BRVO and 6 patients with cataract (non-BRVO) was collected. A proteomic approach which included 2-dimensional electrophoresis (2-DE) coupled with mass spectrometry (MS) and bioinformatics analysis were used to identify $\mathrm{AH}$ proteins with altered expression in patients with macular edema due to BRVO compared with the controls. An enzyme-linked immunosorbent assay was used to validate the proteomic results. The total protein concentration in the AH of patients with BRVO-induced macular edema was significantly greater than that of the controls. In the patients with BRVO, a total of 56 protein spots were significantly altered on the 2D gels. A total of 49 protein spots were identified by MS; many of these proteins have been implicated in angiogenesis, oxidative stress and collagen synthesis. In conclusion, the protein composition in $\mathrm{AH}$ differed significantly between the patients with BRVO and the controls. The identified proteins may be potential biomarkers for the development of macular
\end{abstract}

Correspondence to: Professor Qinghuai Liu, Department of Ophthalmology, First Affiliated Hospital of Nanjing Medical University, 300 Guangzhou Road, Nanjing, Jiangsu, 210029, P.R. China

E-mail: liuqh@njmu.edu.cn

*Contributed equally

Key words: branch retinal vein occlusion, macular edema, aqueous humor, proteomic analysis, matrix-assisted laser desorption ionization time-of-flight/time-of-flight mass spectrometry edema due to BRVO and may play a role in the mechanisms responsible for it.

\section{Introduction}

Branch retinal vein occlusion (BRVO) is the second most common cause of retinal vascular abnormality after diabetic retinopathy and a frequent cause of visual loss (1). In a pooled analysis using existing data from 11 individual populationbased studies, the prevalence of BRVO was found to be 4.42 per 1,000 individuals $(95 \%$ CI 3.65, 5.19). The prevalence of BRVO is greater in Asians (2,3). Visual loss in BRVO, either short or long term, may be the result of the presence of macular edema, macular non-perfusion, retinal neovascularization, vitreous or intraretinal hemorrhage, tractional retinal detachment or a combination of these disorders (4). Macular edema is the most frequent cause of visual impairment in patients with BRVO (5). Thus, understanding the cellular and molecular factors that underlie the pathogenesis of macular edema with BRVO is of critical importance.

The aqueous humor (AH) is an important intraocular fluid responsible for the supply of nutrients to and the removal of metabolic wastes from the avascular tissues of the eye. It is known that protein levels in $\mathrm{AH}$ are altered in various eye diseases, including anterior and posterior segment disorders. In addition, a number of studies have demonstrated that some proteins whose expression is altered in $\mathrm{AH}$ correlate with the mechanisms or prognosis of several eye disorders (6). A number of cytokines and other factors in the $\mathrm{AH}$ have been suggested to be involved in the pathogenesis of macular edema due to BRVO, such as vascular endothelial growth factor (VEGF) and interleukin-6 (IL-6) (5). However, the pathogenesis of macular edema with BRVO is complex; thus, the measurement of these cytokines may not provide enough information as to the disease process. A comprehensive list of the proteins whose expression is altered in the $\mathrm{AH}$ of patients with macular edema due to BRVO is still lacking.

Proteomic analysis is a valuable method for elucidating the molecular nature of $\mathrm{AH}$ (7). High resolution 2-dimensional (2D) polyacrylamide gel electrophoresis (PAGE) is a technique used for the analysis of several hundred proteins in tissues, fluids or cells using only a few microliters of sample and is therefore ideal for analyzing limited volumes of AH. Some researchers have used this technology to explore the patho- 
genesis of various eye diseases (6,8-12). In this study, we used proteomics as a means to identify disease-specific proteins in AH. Through comparative analyses of the proteomes in patients with cataract (controls) and those with macular edema due to BRVO, it may be possible to obtain a better understanding of the molecular events involved in the development of macular edema due to $\mathrm{BRVO}$ and to generate essential data required for the identification of novel biomarkers and/or treatments. The proteomic techniques used include protein separation by 2-DE and characterization by mass spectrometry (MS) of peptides, amino acid sequencing and bioinformatics analysis. Enzymelinked immunosorbent assay (ELISA) was used to validate the results of proteomics.

\section{Materials and methods}

Patients and controls. Twelve AH samples were included in this study, 6 from patients with BRVO-induced macular edema (mean age, $53 \pm 4.98$ years; 3 males and 3 females) and 6 from age-matched patients with cataract without BRVO (mean age, $53.5 \pm 2.35$ years; 3 males and 3 females). The disease course was between 6 and 16 months (mean, $10 \pm 3.406$ months). Clinical data from the patients are summarized in Table I.

The study followed the tenets of the Declaration of Helsinki, and informed written consent was obtained from all patients and controls after we explained the nature and possible consequences of the study. The protocol for this research project was approved by the Ethics Committee of the First Affiliated Hospital of Nanjing Medical University, Nanjing, China.

All participants went through a standard examination including best-corrected visual acuity, slit lamp biomicroscopy, optical coherence tomography (OCT), fundus photo, and fluorescein angiography (FFA). The presence of macular edema was confirmed with FFA and OCT in all patients. No patient had been treated previously for BRVO. None of the controls had any eye diseases other than cataract.

In both groups of examined patients (controls and BRVO), a certain degree of cataract was present. Patients with severe cataract determining blindness or unacceptable vision were not included in the control group.

Sample collection. AH samples were obtained from the eyes of patients with BRVO just before an intravitreal injection of bevacizumab (Avastin, treatment for macular edema due to BRVO) was administered. All sample collections were performed using a standard sterilization procedure as previously described (12). A mean volume of $100 \mu 1$ of AH was collected by anterior chamber limbal paracentesis with a 27-gauge needle attached to an insulin syringe. The intravitreal injection of bevacizumab was then administered through the pars plana. Antibiotic ointment was administered after surgery for 4 days. Immediately after collection, the AH samples were transferred to sterile plastic tubes and stored at $-80^{\circ} \mathrm{C}$ until analysis.

AH samples from patients before cataract surgery were obtained for this study. AH samples from 6 controls (cataract patients without other eye diseases) were also collected as previously described (8). A total of 100-200 $\mu \mathrm{l}$ of sample from each patient sample was pooled. All samples were stored at $-80^{\circ} \mathrm{C}$ until analysis.
Table I. Data from patients with BRVO and the controls.

\begin{tabular}{|c|c|c|c|c|c|}
\hline & No. & $\begin{array}{c}\text { Age } \\
\text { (years) }\end{array}$ & Gender $^{\mathrm{a}}$ & $\begin{array}{c}\text { Course } \\
\text { of disease } \\
\text { (months) }\end{array}$ & $\begin{array}{c}\text { Baseline } \\
\text { central macular } \\
\text { thickness }(\mu \mathrm{m})\end{array}$ \\
\hline \multirow[t]{6}{*}{ Patients } & 1 & 56 & $\mathrm{~F}$ & 16 & 496 \\
\hline & 2 & 47 & $\mathrm{~F}$ & 6 & 523 \\
\hline & 3 & 60 & M & 10 & 457 \\
\hline & 4 & 48 & $\mathrm{~F}$ & 8 & 539 \\
\hline & 5 & 52 & M & 11 & 431 \\
\hline & 6 & 55 & $\mathrm{M}$ & 9 & 511 \\
\hline \multirow[t]{6}{*}{ Controls } & 1 & 56 & M & & \\
\hline & 2 & 52 & $\mathrm{~F}$ & & \\
\hline & 3 & 50 & M & & \\
\hline & 4 & 54 & M & & \\
\hline & 5 & 56 & $\mathrm{~F}$ & & \\
\hline & 6 & 53 & F & & \\
\hline
\end{tabular}

${ }^{\mathrm{a} F}$, female; M, male.

Sample preparation. AH samples from patients or controls were pooled to ensure there was sufficient protein in the extracts for matrix-assisted laser desorption ionization time-of-flight/ time-of-flight mass spectrometry (MALDI-TOF/TOF MS).

Excess salts followed by precipitation of proteins using the ProteoExtract ${ }^{\mathrm{TM}}$ Protein Precipitation kit (Calbiochem, San Diego, CA, USA) were removed in each of the pooled samples. The samples were processed according to the manufacturer's instructions. The protein concentrations of the $\mathrm{AH}$ samples were determined using the Bradford method (Bio-Rad Protein Assay; Bio-Rad, Hercules, CA, USA).

2-DE. 2-DE was performed as previously described (13-15). Twenty-four centimeter, $\mathrm{pH}$ 4-7, NL IPG strips (Amersham Bioscience, Uppsala, Sweden) were rehydrated with $80 \mu \mathrm{g}$ solubilized protein (for silver staining) in a rehydration buffer. After isoelectric focusing, the IPG strips were equilibrated. They were then loaded onto pre-cast $12.5 \%$ homogeneous polyacrylamide gels for electrophoresis, ran in an Ettan-Dalt II system (Amersham Biosciences, San Francisco, CA, USA) and visualized.

Image analysis. The stained gels were scanned and the resulting images were analyzed using ImageMaster ${ }^{\mathrm{TM}}$ 2D Platinum software (version 5.0, Amersham Bioscience, Swiss Institute of Bioinformatics, Geneva, Switzerland) for spot detection, quantification, comparison and analyses, as previously described $(12,13)$. The relative intensities of the spots were used for a comparison between the BRVO and control groups. The statistical comparisons between the intensity of the control and the BRVO protein spots were conducted using the Student's t-test (ImageMaster ${ }^{\mathrm{TM}}$ 2D platinum software, with $\mathrm{p}<0.05$ considered to be significant). The commonly differentially expressed spots (2-fold increase or decrease) were further identified by MALDI-TOF/TOF MS.

Protein identification. Protein identification was performed as previously described $(12,14,16,17)$. In brief, the common differ- 
entially expressed protein spots were excised and the proteins within were reduced, alkylated and digested with trypsin. Digests were immediately spotted onto $600 \mu \mathrm{m}$ anchorchips (Bruker Daltonics, Bremen, Germany). The Bruker Peptide Calibration Mixture was spotted for external calibration. MALDI-TOF MS and tandem TOF/TOF MS were carried out on a time-of-flight Ultraflex II mass spectrometer (Bruker Daltonics). Using the MASCOT search engine [http://www. matrixscience.com; Database: NCBInr 20100409 (10,820,686 sequences; 3,689,795,467 residues); Taxonomy: Homo sapiens (human) (231,301 sequences)] based on the Swiss-Prot protein database, peptide mass fingerprinting was performed for the identification of proteins from tryptic fragment sizes using the assumption that peptides are monoisotopic. One missed trypsin cleavage was allowed. A mass tolerance of 100 parts per million (ppm) was the window of error allowed for matching the peptide mass values.

Gene Ontology analysis. All the proteins identified in this experiment were subjected to Gene Ontology (www.geneontology.org/) for molecular function, biological process and cellular component analysis.

ELISA. Concentrations of alpha crystallin A chain (CRYAA) in the AH samples were verified and quantified using commercially available human cytokine ELISA kits from Uscn Life Science Inc. (Catalog no. E9662h; Wuhan, China). The recommended protocol of the manufacturer was followed in all cases. Briefly, standards and AH samples were added to antibody-coated 96-well plates and incubated for $2 \mathrm{~h}$ at room temperature, followed by the addition of biotin-conjugated polyclonal antibody specific for CRYAA and incubation for an additional $1 \mathrm{~h}$. The plates were then washed and incubated with avidin conjugated to horseradish peroxidase for $1 \mathrm{~h}$ at $37^{\circ} \mathrm{C}$. Subsequently, a tetramethylbenzidine substrate solution was added to each well. The enzyme-substrate reaction was terminated by the addition of sulfuric acid solution. The color change was measured by spectrophotometry at a wavelength of $450 \mathrm{~nm}$. A standard curve was plotted from measurements made with the standard solution (from 0.78 to $50 \mathrm{ng} / \mathrm{ml}$ for CRYAA) and was used to determine the concentration of CRYAA in each sample. The concentration of CRYAA in the samples was determined by comparing the OD of the samples to the standard curve. All measurements were performed in duplicate. CRYAA concentrations were calculated as per nanogram of protein.

Statistical analysis. The protein spots were visualized using ImageMaster $^{\mathrm{TM}}$ 2D Platinum software as described in the image analysis section. The variation in protein spot intensity within a sample map and between 2 sample maps was analyzed using the Student's t test. The ELISA results were also analyzed using the Student's t test.

\section{Results}

Protein content in AH from patients with BRVO and controls. A total of $12 \mathrm{AH}$ samples were included in this study, 6 from patients with BRVO and 6 from age-matched cataract patients without BRVO. There was no statistically significant differ-

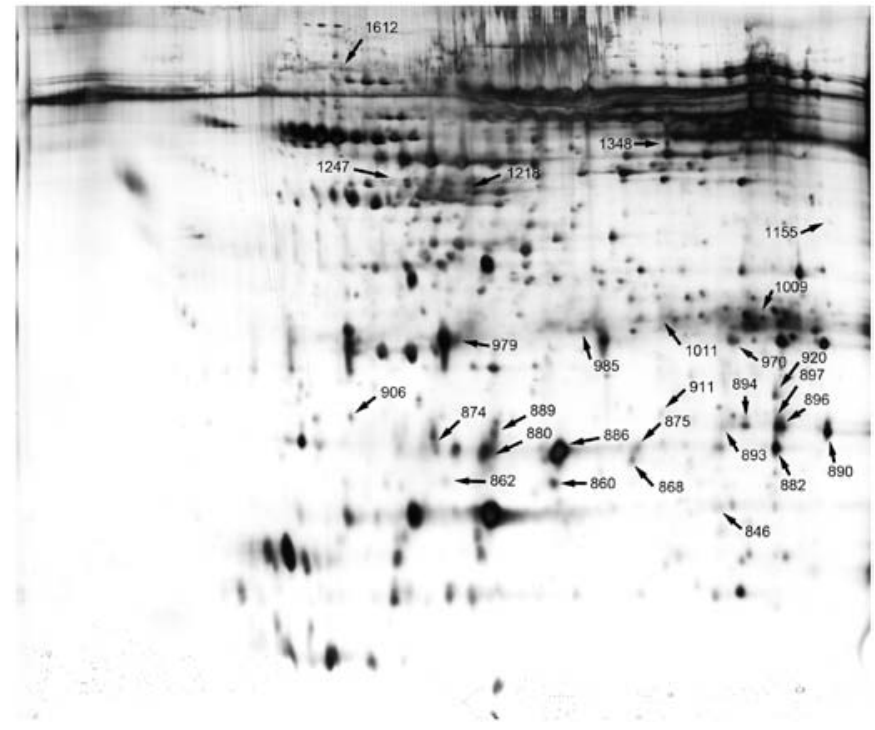

Figure 1. Two-dimensional (2D) gel images of aqueous humor (AH) samples from the controls. The relative spot intensity was calculated based on the spot volume using ImageMaster ${ }^{\mathrm{TM}} 2 \mathrm{D}$ platinum software and the labels indicate proteins whose expression was altered by a factor of $>2$ in the control group. Only the spots labeled with numbers were used for MALDI-TOF/TOF MS/ MS analysis.

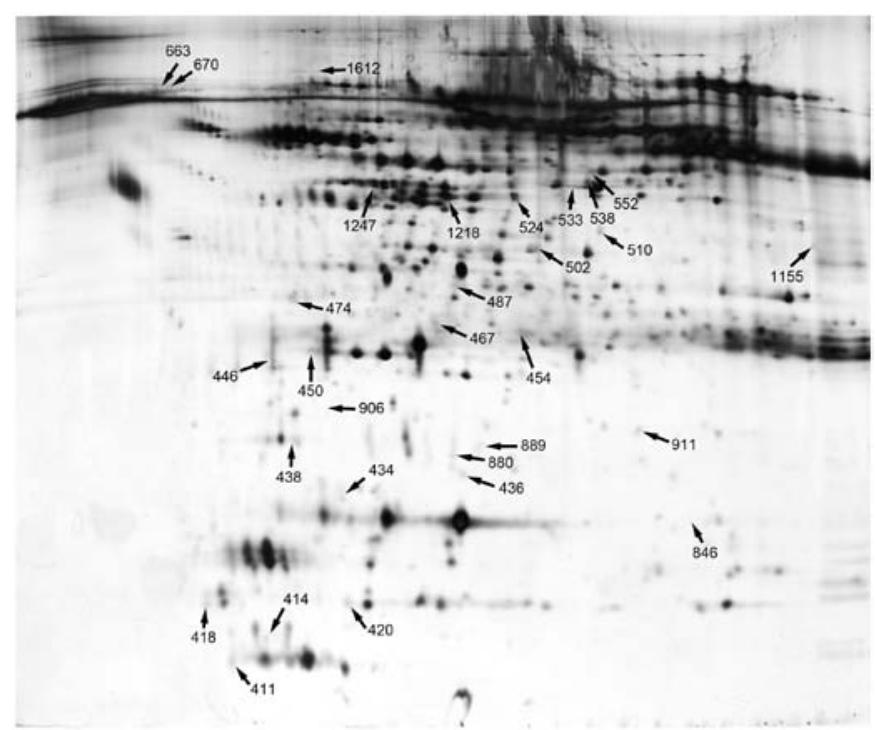

Figure 2. Two-dimensional (2D) gel images of aqueous humor (AH) samples from patients with BRVO. The relative spot intensity was calculated based on the spot volume using ImageMaster ${ }^{\mathrm{TM}} 2 \mathrm{D}$ platinum software and the labels indicate proteins whose expression was altered by a factor of $>2$ in the branch retinal vein occlusion (BRVO) group. Only the spots labeled with numbers were used for MALDI-TOF/TOF MS/MS analysis.

ence between the 2 groups as regards age $(\mathrm{p}=0.074)$. Clinical data from the patients are summarized in Table I.

The mean total protein level in $\mathrm{AH}$ from patients with BRVO was $1.124 \mathrm{mg} / \mathrm{ml}$, while that from the controls was $0.545 \mathrm{mg} / \mathrm{ml}$. Total protein levels in the patients with BRVO were significantly greater than those of the controls.

2-DE patterns. Figs. 1 and 2 depict the 2D gel images from patients and the controls. Gel images from patients with BRVO displayed more spots and more intensely silver stained spots than 


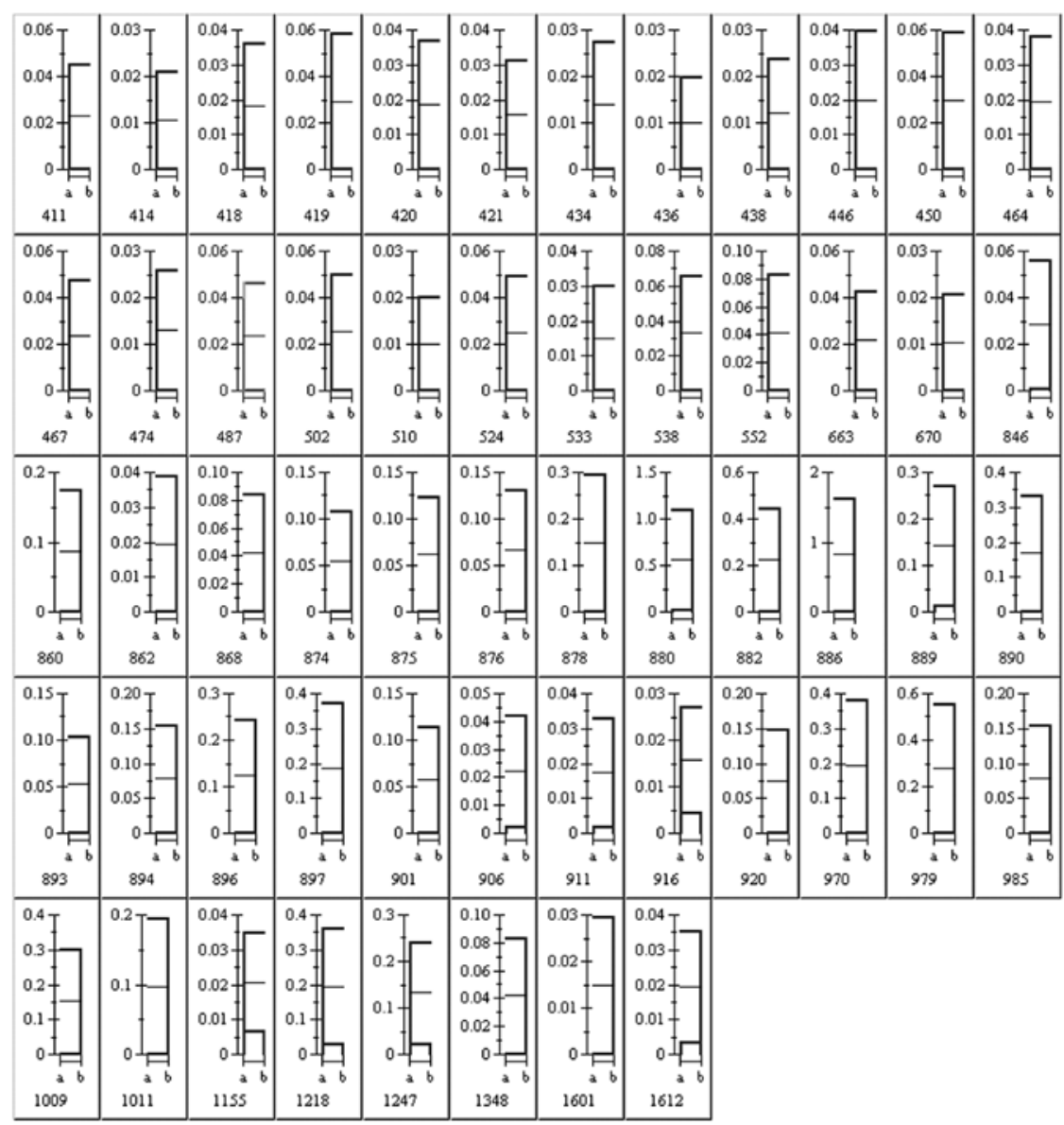

Figure 3. Comparing the relative intensities of spots between the branch retinal vein occlusion (BRVO) and the control groups. The stained gels were scanned, and resulting images were analyzed using the ImageMaster ${ }^{\mathrm{TM}}$ 2D Platinum software for spot detection, quantification, comparison and analyses. The relative intensities of spots were used for comparison between the BRVO and control groups. A total of 56 spots were altered by $>2$-fold in 2-dimensional (2D) gels from patients with BRVO-induced macular edema.

the gel images from the controls. There were significant differences in relative spot volumes (\% volume) in the gel patterns; patients with BRVO showed greater volumes than the controls. The stained gels were scanned and the resulting images were analyzed using ImageMaster ${ }^{\mathrm{TM}}$ 2D Platinum software for spot detection, quantification, comparison and analyses. A total of 56 protein spots were altered by $>2$-fold in the $2 \mathrm{D}$ gels from patients with BRVO-induced macular edema (Fig. 3).

Identification of proteins. Based on the results presented above, 56 protein spots were isolated for further analysis. Each spot was acquired from the gel and digested extensively with trypsin. The resulting peptides were applied to a MALDI TOF/TOF MS for measurements. A total of 49 protein spots were identified by MS, including fibroblast growth factor- 4 (FGF-4), hepatoma-derived growth factor (HDGF) and crystallins. Many of these proteins have been implicated in angiogenesis, oxidative stress and collagen synthesis. The identified proteins [name, function, molecular weight (MW), isoelectric point (PI) and sequence coverage] are listed in Table II.

Gene Ontology analysis. All the proteins identified in this study were subjected to Gene Ontology (www.geneontology. org/) for molecular function, biological process and cellular component analysis. The results are listed in Table II.

Aqueous levels of CRYAA in BRVO patients and controls. Aqueous levels of CRYAA in the AH of BRVO patients or controls were below the minimum detectable concentration (data not shown).

\section{Discussion}

BRVO is a common cause of retinal vascular abnormality and a frequent cause of visual loss. Macular edema is the most frequent cause of visual impairment in patients with BRVO. Therefore, understanding the cellular and molecular factors that underlie the pathogenesis of macular edema with BRVO is of particular importance. However, the majority of studies on BRVO have focused on the treatment and only a few studies have emphasized the pathogenesis of macular edema due to BRVO $(5,18-22,26)$.

To assess the severity of macular edema with BRVO by obtaining a sample of the AH or vitreous fluid at surgery is of critical importance. Several cytokines and other factors in the ocular fluid have been suggested to be involved in the pathogenesis of macular edema due to BRVO, such as VEGF and 


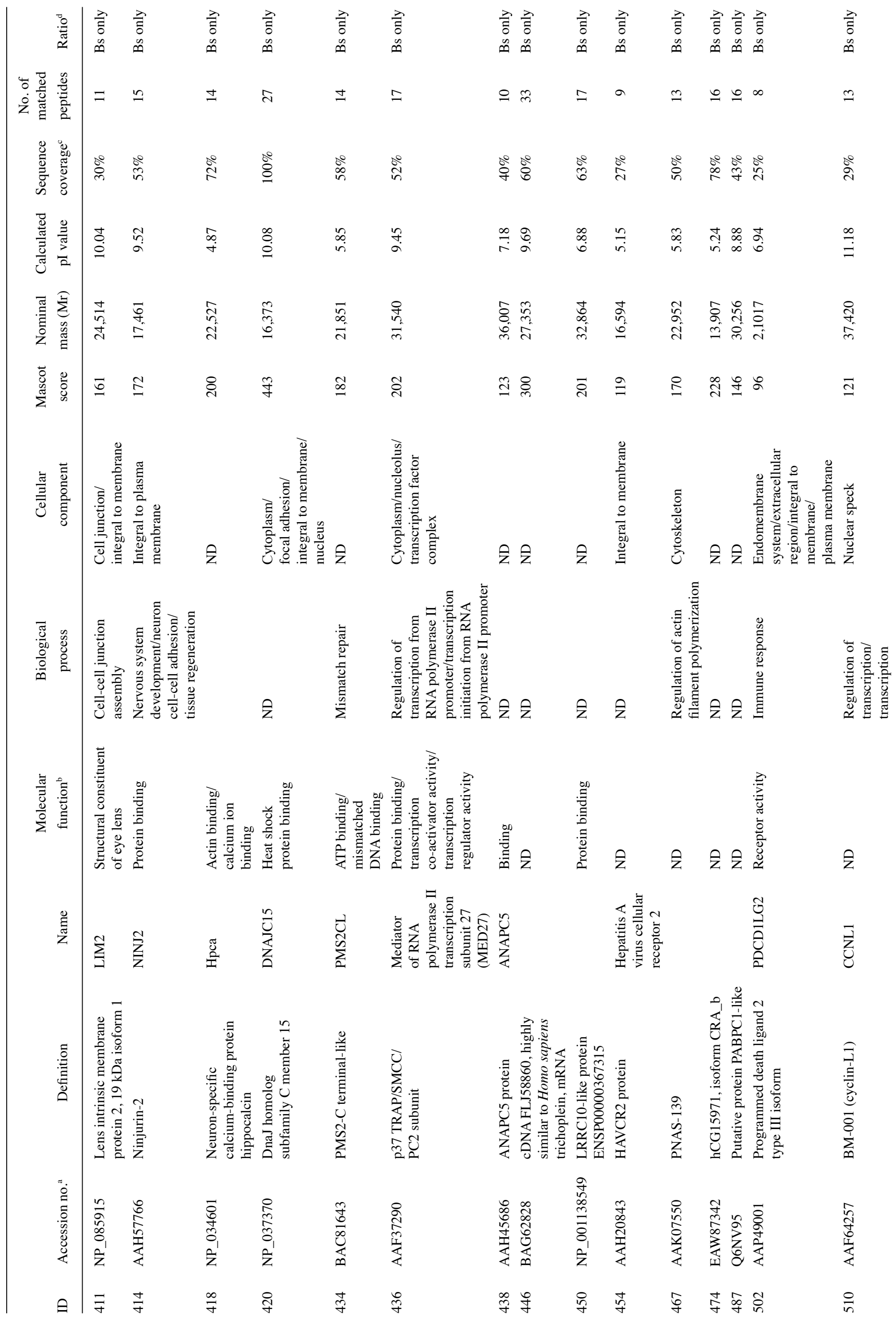




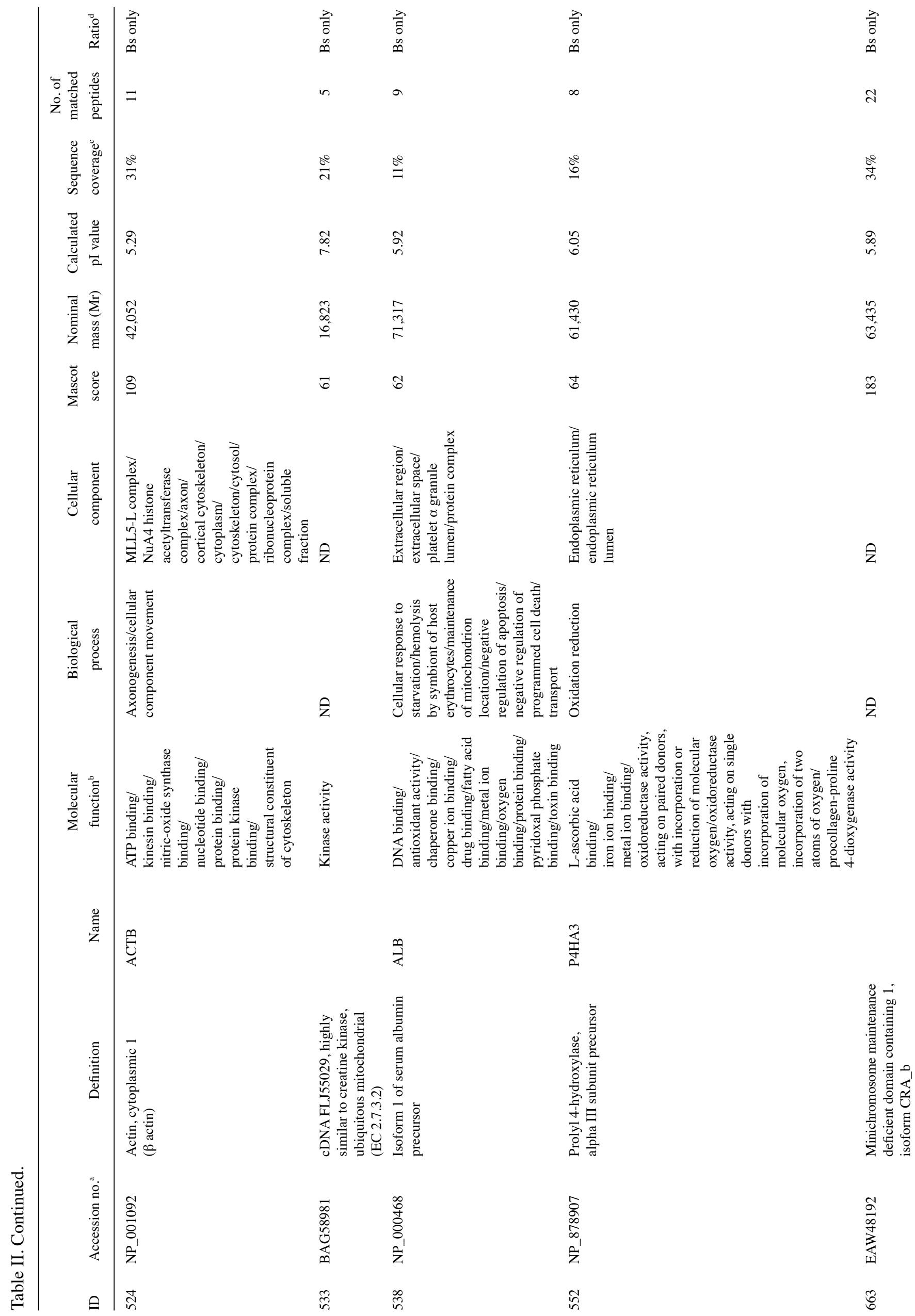




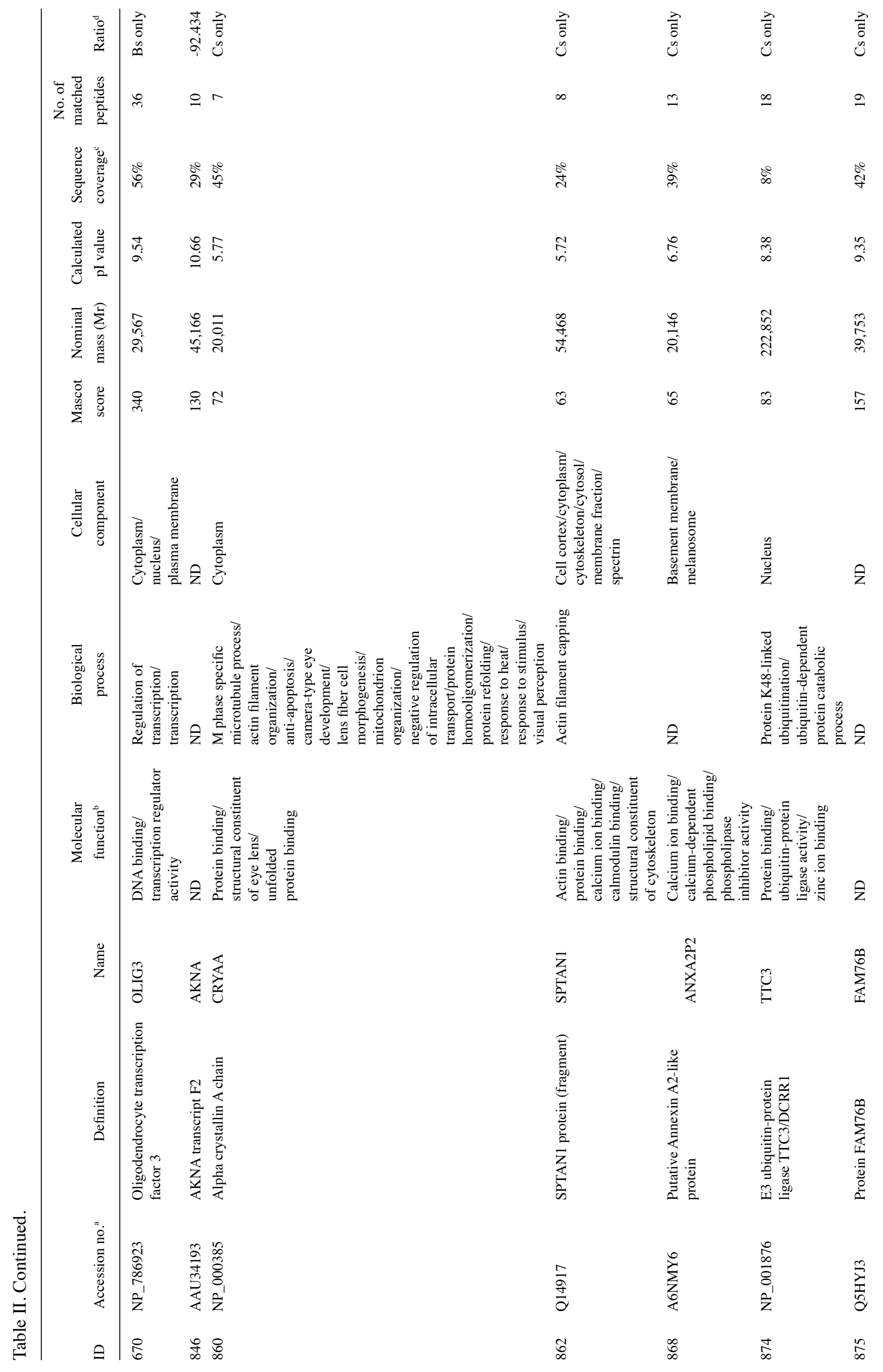




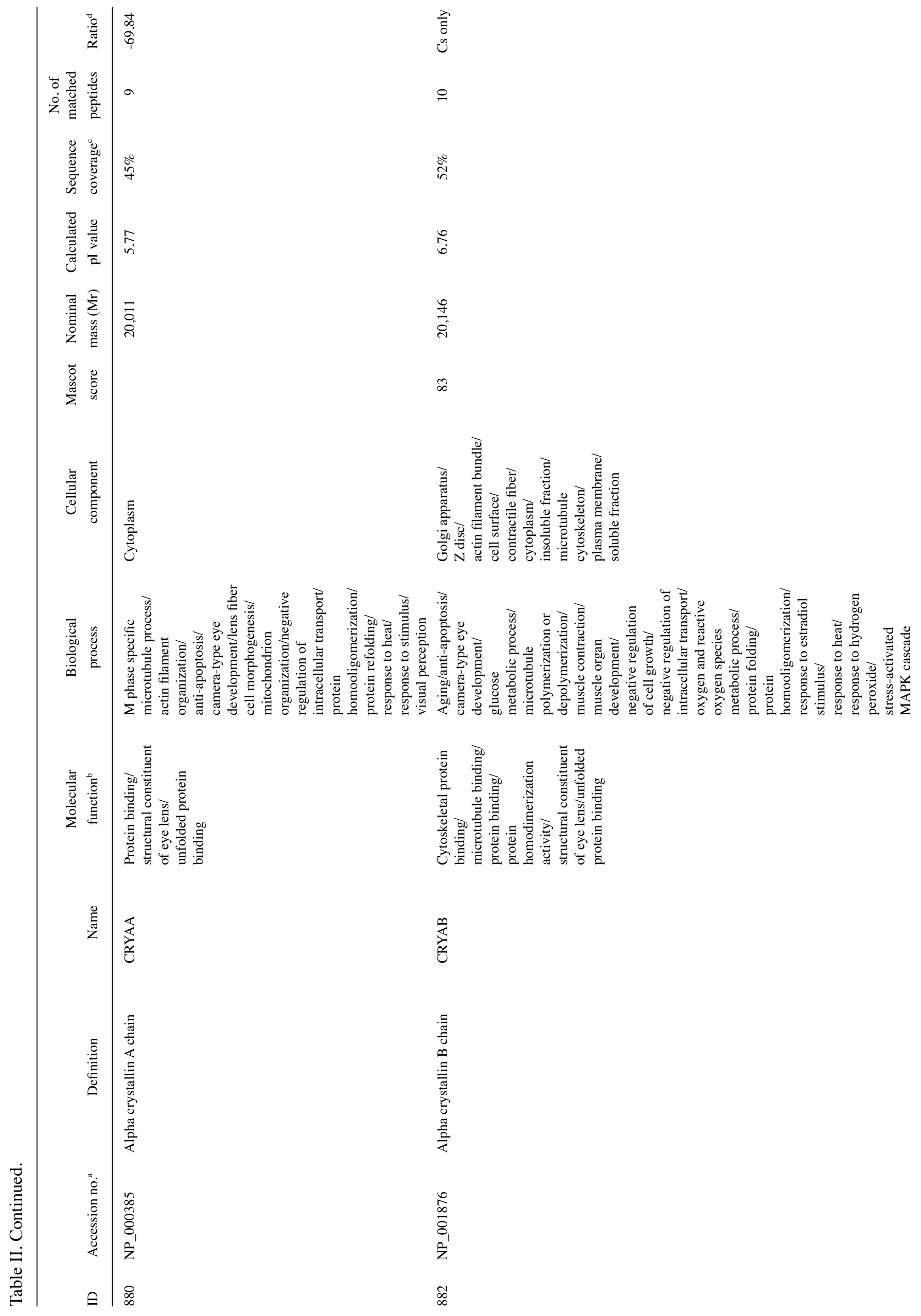




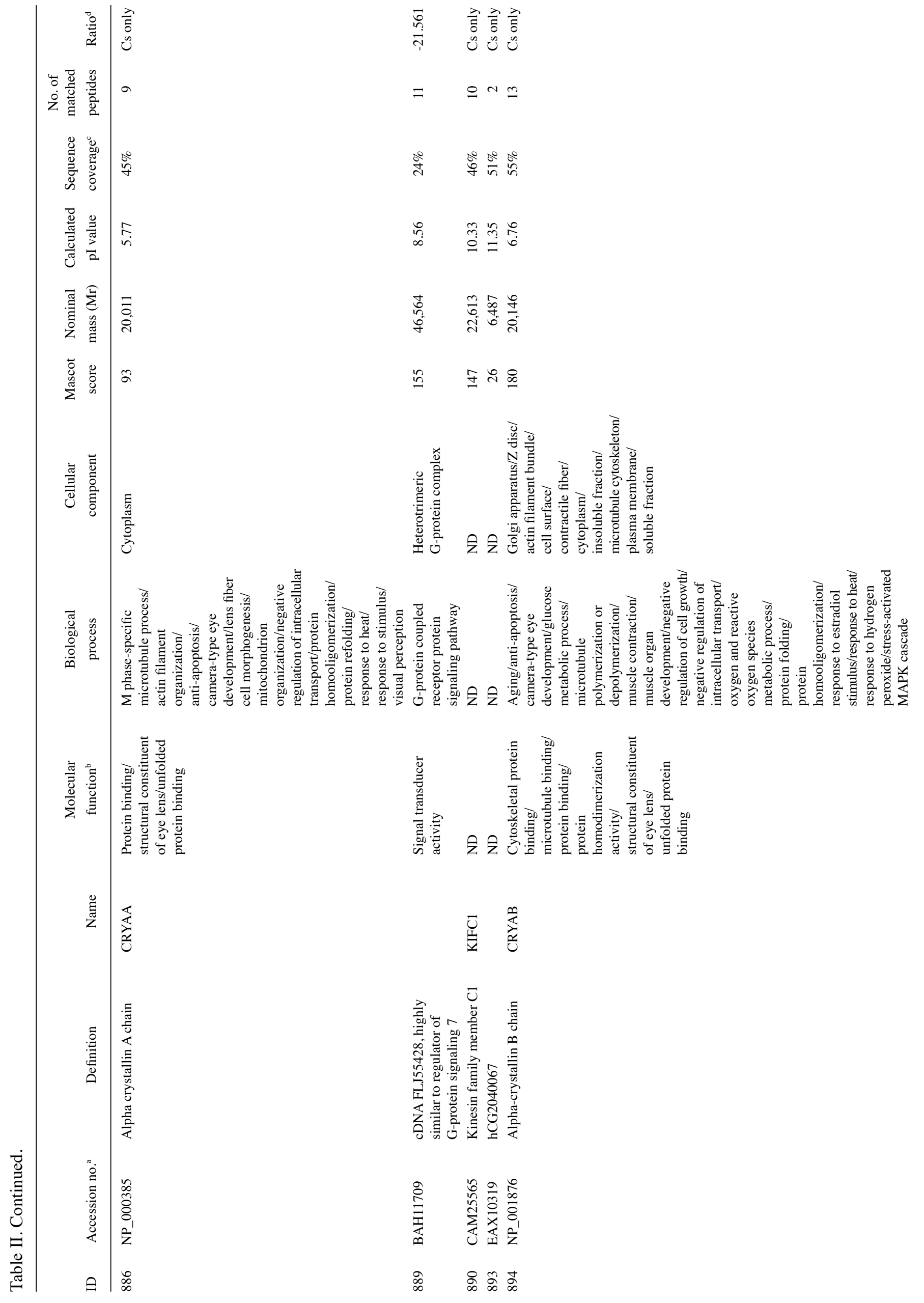




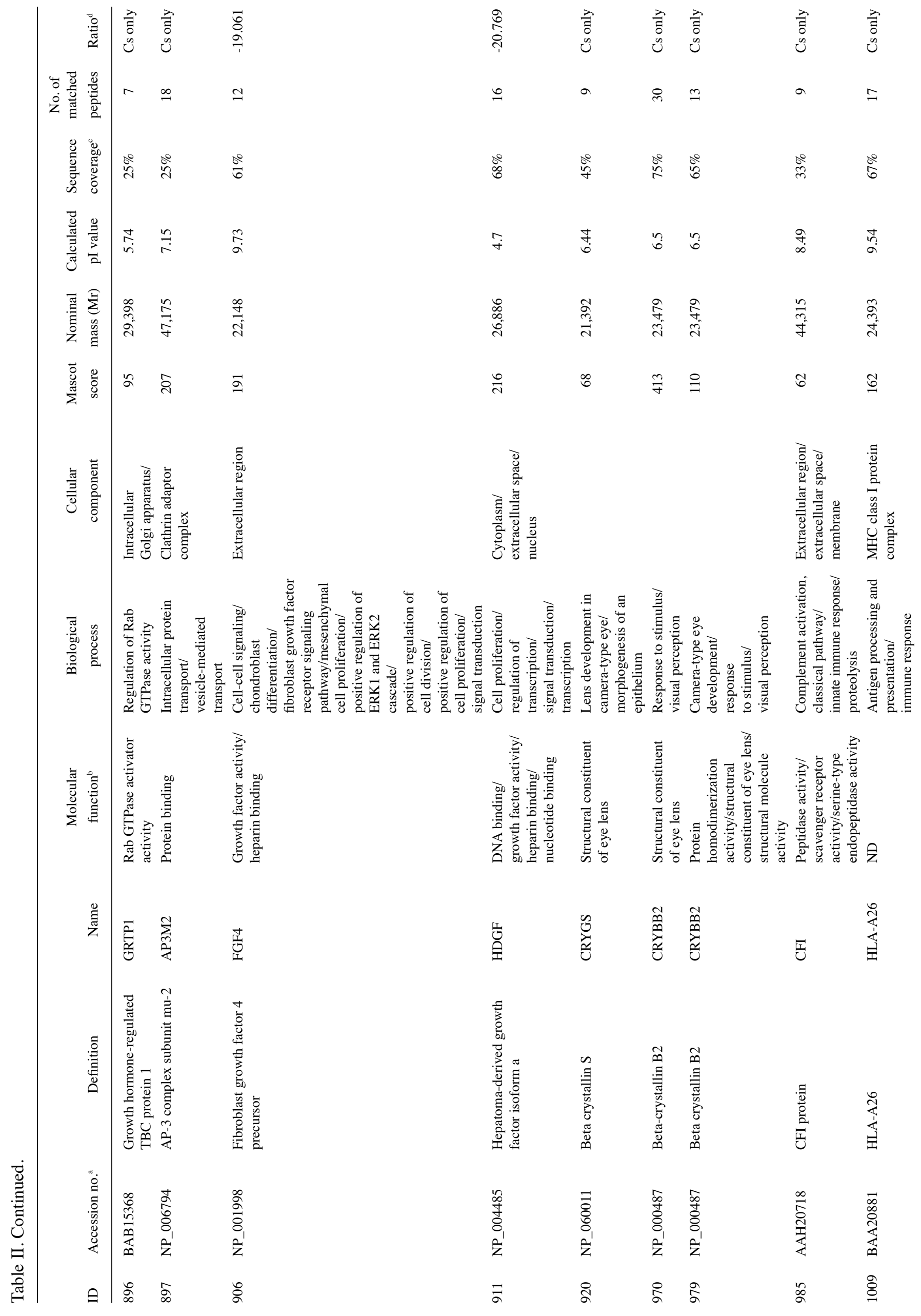




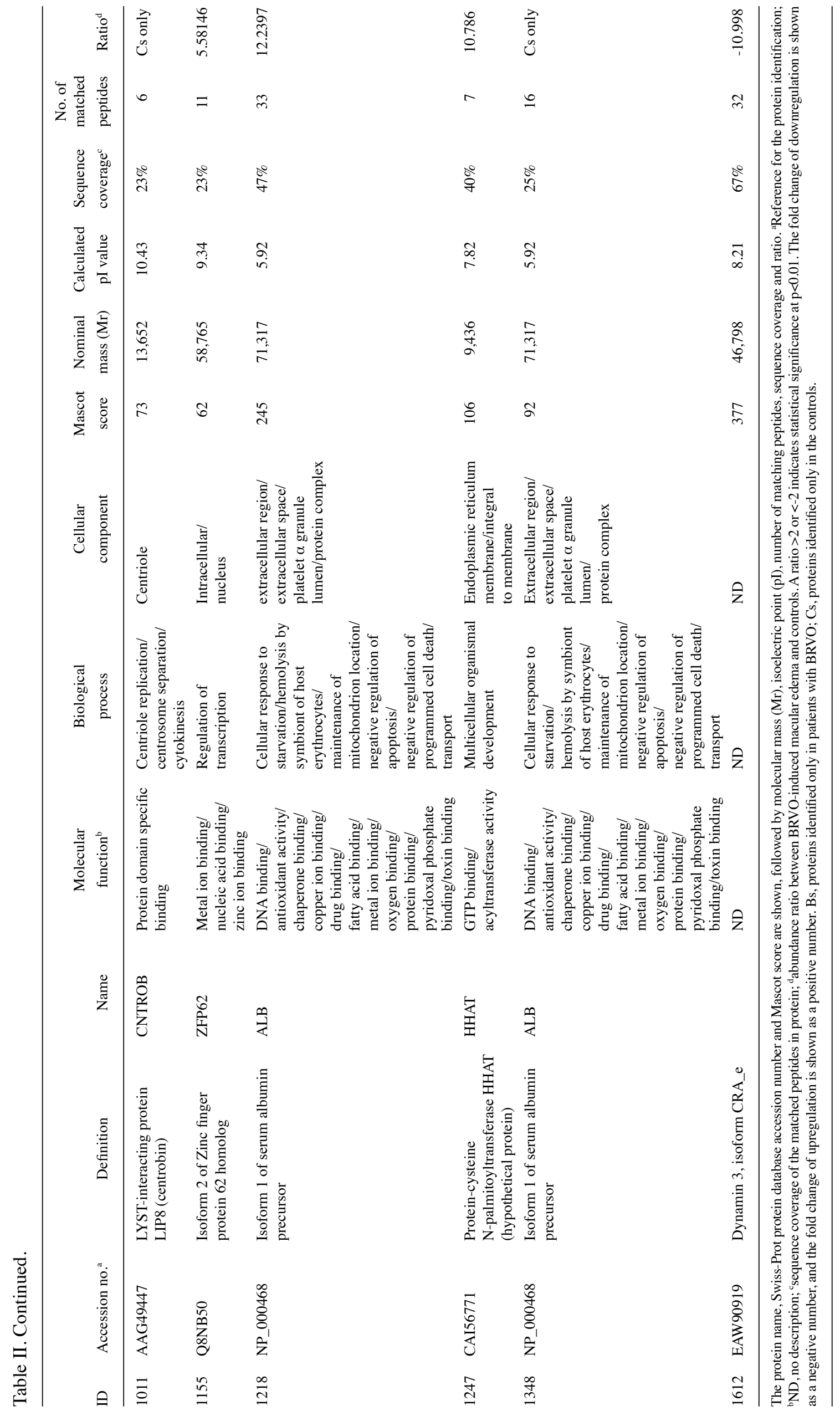


IL-6 $(5,18,19)$. However, the surgical harvesting of vitreous fluid is associated with the risk of vitreous haemorrhage, retinal tears and retinal detachment, whereas it is difficult to obtain vitreous samples for diagnostic or investigative purposes without performing surgery. On the other hand, obtaining $\mathrm{AH}$ samples is a far easier and less risky procedure. AH can be collected directly from patients and $\mathrm{AH}$ proteins may manifest discrete changes in patients with BRVO.

To date, only a few studies have examined the changes in $\mathrm{AH}$ protein epxression in patients with BRVO. The exact changes in protein expression that occur in $\mathrm{AH}$ in patients with BRVO are unclear. Therefore, it was considered of importance to study the changes in $\mathrm{AH}$ protein expression in patients with BRVO. Such information may provide new insight into the mechanisms of BRVO and identify potential biomarkers of this condition. $\mathrm{AH}$ is valuable for understanding eye disorders and certain studies have tried to identify the majority of the proteins in AH of patients with cataract (7,23-25). Previous studies have suggested that $\mathrm{AH}$ proteins activate signaling cascades, which subsequently regulate cellular functions, including mitosis, differentiation, motility, apoptosis and angiogenesis. Such proteins may play a vital role in the pathology of macular edema secondary to BRVO. Noma et al found that the aqueous level of VEGF reflected its vitreous level (26); thus, in this study, we investigated the pathogenesis of macular edema induced by BRVO by measuring alterations in protein expression in $\mathrm{AH}$ samples from patients with BRVO.

Proteomic analysis is a valuable method for elucidating the molecular nature of $\mathrm{AH}$. Some studies have used this technology to explore the pathogenesis of various eye diseases $(6,8-12)$. In this study, we conducted proteomic analysis of $\mathrm{AH}$ from patients with macular edema induced by BRVO and agematched patients with cataract (controls). The abnormal expression and distribution of proteins in $\mathrm{AH}$ were identified. The patterns of 2-DE gels in the patients with BRVO differed from the controls, which indicated that the protein content in the AH changes with the development of BRVO. Proteomics revealed that 56 protein spots were altered by $>2$-fold, which suggests that there are complex mechanisms involved in the pathogenesis of BRVO.

Several of the proteins identified by proteomics have been implicated in angiogenesis, oxidative stress and collagen synthesis. Such proteins may play a vital role in the pathogenesis of macular edema induced by BRVO.

FGF-4 was found to be downregulated in this study. FGF-4 is a member of the fibroblast growth factor family and it induces the proliferation, migration and survival of several cell types, including endothelial cells. FGF-4 induces vascular permeability, therapeutic angiogenesis and arteriogenesis comparable to that of VEGF (27). Other results point to an indirect angiogenic activity of FGF-4 through the autocrine induction of VEGF secretion $(28,29)$. In a previous study, the induction of the angiogenic morphotype and the parallel modulations of the biosynthetic phenotype in human umbilical vein endothelial cells were completely suppressed by a neutralizing antibody directed against VEGF (28).

HDGF was found to be downregulated in this study, which is mitogenic for vascular smooth muscle and aortic endothelial cells. HDGF is a highly expressed vascular endothelial cell protein in vivo and is a potent endothelial mitogen and regulator of endothelial cell migration by mechanisms distinct from VEGF. As previously demonstrated, with the chick chorioallantoic membrane (CAM), a bioassay for angiogenesis, exogenous recombinant HDGF significantly stimulated blood vessel formation and a dose-dependent reorganization of cells within the CAM into a more compact, linear alignment reminiscent of tube formation (30).

Some crystallins were identified to be downregulated in this study. Alpha crystallins are chaperones belonging to the small heat shock protein family. Certain studies have suggested that the expression of alphaA and alphaB crystallin is related to oxidative stress $(31,32)$. AlphaB-crystallin (CRYAB) plays an important role as a chaperone for VEGF-A in angiogenesis. The attenuation of intraocular angiogenesis has been observed in CRYAB knockout [CRYAB (-/-)] mice in 2 models of intraocular disease: oxygen-induced retinopathy and laser-induced choroidal neovascularization. VEGF-A protein expression was low in CRYAB (-/-) mouse retinas compared with wild-type mouse retinas. CRYAB (-/-) retinal pigment epithelial (RPE) cells showed low VEGF-A secretion under serum-starved conditions compared with wild-type cells. CRYAB can bind to VEGF-A but not transforming growth factor- $\beta$ in cultured RPE cells. CRYAB and VEGF-A are co-localized in the endoplasmic reticulum in RPE cells under chemical hypoxia. Endothelial cell apoptosis in newly formed vessels was greater in CRYAB (-/-) than wild-type mice (33). Ghosh et al found that human CRYAB peptides have strong interactions with FGF-2 and VEGF, which are both related with angiogenesis. Chaperone assays confirmed the ability of CRYAB to protect against the aggregation of FGF-2 and VEGF (34). $\alpha$ - and $\beta$-crystallin isoforms are overexpressed with diabetes, as shown by proteomics and confirmed by immunoblotting (35). These data suggest that crystallins may function together with VEGF during angiogenesis.

Complement factor I (CFI) was also downregulated in our study. In a previous study, CFI was found to be increased in proliferative diabetic retinopathy vitreous compared with non-diabetic vitreous by a comprehensive proteomic analysis [one-dimensional SDS-PAGE and nano-liquid chromatography (LC)/MS/MS] (36). Thus, CFI may also play a role in angiogenesis.

FGF-4, platelet-derived growth factor (PDGF), crystallins and CFI, which are tightly associated with angiogenesis, were all downregulated in this study. This may be due to the fact that the patients in this study had a long course of disease and the angiogenic tissues had become quiescent. Further investigations are required.

Albumin, anaphase promoting complex subunit 5 (ANAPC5) and $\beta$-actin were found to be all upregulated in this study. Human serum albumin (HSA) is the most abundant protein in the circulatory system, and one of its principal functions is to transport fatty acids (37). Albumin is also a very abundant and important circulating antioxidant (38). HSA inhibits endothelial apoptosis in a highly specific manner (39). Increased vascular disease occurs with low albumin, possibly reflecting the specific inhibition of endothelial apoptosis reported for tissue culture (40). Serum albumin has been significantly associated with the severity of retinopathy and neuropathy in patients with type 2 diabetes (41). It has been reported that the expression of ANAPC5 may represent an 
important event in the pathogenesis of vascular proliferative diseases (42).

Actins are highly conserved proteins that are involved in cell motility, structure and integrity. $\beta$-actin is a major constituent of the contractile apparatus and one of the two non-muscle cytoskeletal actins. $\beta$-actin as a transcription factor also stimulates endothelial nitric oxide synthase (eNOS) expression (43).

Of note, some proteins identified in this study may participate in collagen synthesis, the cytoskeleton and organization of the actin cytoskeleton, such as prolyl 4-hydroxylase, alpha polypeptide III (P4HA3); spectrin, alpha, non-erythrocytic 1, alpha-fodrin (SPTAN1); actin related protein 2/3 complex, subunit 2, $34 \mathrm{kDa}$ (ARPC2, also known as PNAS-139). These events are critical for driving a wide range of cellular processes, including motility, endocytosis and intracellular trafficking. Previous studies have demonstrated that the level of IL-6 is increased in the AH of diabetic patients with macular edema and in patients with macular edema inducedy by BRVO $(5,44)$. IL- 6 can induce an increase in endothelial permeability in vitro by rearranging actin filaments and by changing the shape of endothelial cells (45). Thus, actin filaments may participate in the pathogenesis of macular edema due to BRVO.

Other proteins identified play crucial roles in photoreceptor or retina functions. Some proteins identified in this study may control cell cycle progression and/or apoptosis, immune responses and oxidative stress. Further investigations are rquired to fully understand the exact association between changes in $\mathrm{AH}$ protein expression and BRVO.

Although a comparison of AH samples from patients with BRVO with and without macular edema may be more useful to analyze the proteins in patients with BRVO that may be involved in the development of macular edema, it is not easy to obtain AH samples from patients with BRVO without macular edema. Moreover, AH from normal healthy adults cannot be obtained ethically. Therefore, we used patients with cataract as the controls in this study, as previously done by others $(6,8-11)$. Therefore, it is possible that some of the proteins identified in our study are present due to the underlying cataract condition. However, the patients with BRVO were all senile and agematched with the controls and all had cataract which did not require surgery. Thus, the 2 groups were comparable.

Due to the use of pooled samples, the results in this study do not provide any information as to the variation between patients within a group. Thus, it is possible that patient-to-patient variability exists within the current study. However, the use of pooled samples should reduce the component of patient-to-patient variation and reveal overall differences between patients and controls, as previously described (45). The use of lesser numbers of pooled $\mathrm{AH}$ is a disadvantage in this aspect. The influence of patient-to-patient variation will be addressed in a subsequent study. Our results may prove valuable for future research in the pathogenesis in BRVO.

In conclusion, the results of the present study revealed that the proteomic composition of AH was differed significantly between the patients with macular edema with BRVO and the controls. The proteins identified may serve as potential biomarkers for macular edema induced by BRVO.

\section{Acknowledgements}

This study was supported by grants from the National Basic Research Program of China (973 Program, no. 2011CB510200) and the National Natural Science Foundation of China (no. 81170855).

\section{References}

1. Lim JW: Intravitreal bevacizumab and cytokine levels in major and macular branch retinal vein occlusion. Ophthalmologica 225: $150-154,2011$.

2. Rogers S,McIntosh RL, Cheung N, et al: The prevalence of retinal vein occlusion: pooled data from population studies from the United States, Europe, Asia, and Australia. Ophthalmology 117: 313-319, 2010.

3. Zhou JQ, Xu L, Wang S, et al: The 10-year incidence and risk factors of retinal vein occlusion: the Beijing eye study. Ophthalmology 120: 803-808, 2013.

4. McIntosh RL, Mohamed Q, Saw SM and Wong TY: Interventions for branch retinal vein occlusion: an evidence-based systematic review. Ophthalmology 114: 835-854, 2007.

5. Noma H, Funatsu H, Yamasaki M, et al: Pathogenesis of macular edema with branch retinal vein occlusion and intraocular levels of vascular endothelial growth factor and interleukin-6. Am J Ophthalmol 140: 256-261, 2005.

6. Duan X, Lu Q, Xue P, et al: Proteomic analysis of aqueous humor from patients with myopia. Mol Vis 14: 370-377, 2008.

7. Chowdhury UR, Madden BJ, Charlesworth MC and Fautsch MP: Proteome analysis of human aqueous humor. Invest Ophthalmol Vis Sci 51: 4921-4931, 2010.

8. Richardson MR, Segu ZM, Price MO, et al: Alterations in the aqueous humor proteome in patients with Fuchs endothelial corneal dystrophy. Mol Vis 16: 2376-2383, 2010.

9. Funding M, Vorum H, Honore B, Nexo E and Ehlers N: Proteomic analysis of aqueous humour from patients with acute corneal rejection. Acta Ophthalmol Scand 83: 31-39, 2005.

10. Izzotti A, Longobardi M, Cartiglia C and Sacca SC: Proteome alterations in primary open angle glaucoma aqueous humor. J Proteome Res 9: 4831-4838, 2010.

11. Duan X, Xue P, Wang N, Dong Z, Lu Q and Yang F: Proteomic analysis of aqueous humor from patients with primary open angle glaucoma. Mol Vis 16: 2839-2846, 2010.

12. Yao J, Liu X, Yang Q, et al: Proteomic analysis of the aqueous humor in patients with wet age-related macular degeneration. Proteomics Clin Appl 7: 550-560, 2013.

13. Yao JQ, Liu QH, Chen X, et al: Hsp90 inhibitor 17-allylamino17-demethoxygeldanamycin inhibits the proliferation of ARPE-19 cells. J Biomed Sci 17: 30, 2010.

14. Gong Y, Chen N, Wang FQ, Wang ZH and Xu HX: Serum proteome alteration of severe sepsis in the treatment of continuous renal replacement therapy. Nephrol Dial Transplant 24: 3108-3114, 2009.

15. Sun ZL, Zhu Y, Wang FQ, et al: Serum proteomic-based analysis of pancreatic carcinoma for the identification of potential cancer biomarkers. Biochim Biophys Acta 1774: 764-771, 2007.

16. Wu J, Wang F, Gong Y, et al: Proteomic analysis of changes induced by nonylphenol in Sprague-Dawley rat Sertoli cells. Chem Res Toxicol 22: 668-675, 2009.

17. Su Y, Liu R, Sheng J, et al: Malignant progression in $\mathrm{O}^{6}$-methylguanine-DNA methyltransferase-deficient esophageal cancer cells is associated with Ezrin protein. DNA Cell Biol 31: 856-866, 2012.

18. Noma H, Minamoto A, Funatsu H, et al: Intravitreal levels of vascular endothelial growth factor and interleukin-6 are correlated with macular edema in branch retinal vein occlusion. Graefes Arch Clin Exp Ophthalmol 244: 309-315, 2006.

19. Noma H, Funatsu H, Mimura T and Shimada K: Increase of aqueous inflammatory factors in macular edema with branch retinal vein occlusion: a case control study. J Inflamm (Lond) 7: 44, 2010.

20. Lee WJ, Kang MH, Seong M and Cho HY: Comparison of aqueous concentrations of angiogenic and inflammatory cytokines in diabetic macular oedema and macular oedema due to branch retinal vein occlusion. Br J Ophthalmol 96: 1426-1430, 2012. 
21. Noma H, Funatsu H, Mimura T and Eguchi S: Vascular endothelial growth factor receptor-2 in macular oedema with retinal vein occlusion. Ophthalmic Res 48: 56-58, 2012.

22. Fonollosa A, Garcia-Arumi J, Santos E, et al: Vitreous levels of interleukine- 8 and monocyte chemoattractant protein- 1 in macular oedema with branch retinal vein occlusion. Eye (Lond) 24: 1284-1290, 2010.

23. Bennett KL, Funk M, Tschernutter M, et al: Proteomic analysis of human cataract aqueous humour: Comparison of one-dimensional gel LCMS with two-dimensional LCMS of unlabelled and iTRAQ ${ }^{\circledR}$-labelled specimens. J Proteomics 74: 151-166, 2011.

24. Escoffier P, Paris L, Bodaghi B, Danis M, Mazier D and Marinach-Patrice C: Pooling aqueous humor samples: bias in 2D-LC-MS/MS strategy? J Proteome Res 9: 789-797, 2010.

25. Richardson MR, Price MO, Price FW, et al: Proteomic analysis of human aqueous humor using multidimensional protein identification technology. Mol Vis 15: 2740-2750, 2009.

26. Noma H, Funatsu H, Yamasaki M, et al: Aqueous humour levels of cytokines are correlated to vitreous levels and severity of macular oedema in branch retinal vein occlusion. Eye (Lond) 22: 42-48, 2008

27. Rissanen TT, Markkanen JE, Arve K, et al: Fibroblast growth factor 4 induces vascular permeability, angiogenesis and arteriogenesis in a rabbit hindlimb ischemia model. Faseb J 17: 100-102, 2003.

28. Deroanne CF, Hajitou A, Calberg-Bacq CM, Nusgens BV and Lapiere CM: Angiogenesis by fibroblast growth factor 4 is mediated through an autocrine up-regulation of vascular endothelial growth factor expression. Cancer Res 57: 5590-5597, 1997

29. Hajitou A, Deroanne C, Noel A, et al: Progression in MCF-7 breast cancer cell tumorigenicity: compared effect of FGF-3 and FGF-4. Breast Cancer Res Treat 60: 15-28, 2000.

30. Everett AD, Narron JV, Stoops T, Nakamura H and Tucker A: Hepatoma-derived growth factor is a pulmonary endothelia cell-expressed angiogenic factor. Am J Physiol Lung Cell Mol Physiol 286: L1194-L1201, 2004.

31. Kumar PA, Haseeb A, Suryanarayana P, Ehtesham NZ and Reddy GB: Elevated expression of alphaA- and alphaBcrystallins in streptozotocin-induced diabetic rat. Arch Biochem Biophys 444: 77-83, 2005.

32. Rao NA, Saraswathy S, Wu GS, Katselis GS, Wawrousek EF and Bhat S: Elevated retina-specific expression of the small heat shock protein, alphaA-crystallin, is associated with photoreceptor protection in experimental uveitis. Invest Ophthalmol Vis Sci 49: 1161-1171, 2008.

33. Kase $\mathrm{S}, \mathrm{He} \mathrm{S}$, Sonoda $\mathrm{S}$, et al: alphaB-crystallin regulation of angiogenesis by modulation of VEGF. Blood 115: 3398-3406, 2010.
34. Ghosh JG, Shenoy AK Jr and Clark JI: Interactions between important regulatory proteins and human alphaB crystallin. Biochemistry 46: 6308-6317, 2007.

35. Fort PE, Freeman WM, Losiewicz MK, Singh RS and Gardner TW: The retinal proteome in experimental diabetic retinopathy: up-regulation of crystallins and reversal by systemic and periocular insulin. Mol Cell Proteomics 8: 767-779, 2009

36. Gao BB, Chen X, Timothy N, Aiello LP and Feener EP: Characterization of the vitreous proteome in diabetes without diabetic retinopathy and diabetes with proliferative diabetic retinopathy. J Proteome Res 7: 2516-2525, 2008.

37. Kragh-Hansen U, Watanabe H, Nakajou K, Iwao Y and Otagiri M: Chain length-dependent binding of fatty acid anions to human serum albumin studied by site-directed mutagenesis. J Mol Biol 363: 702-712, 2006.

38. Roche M, Rondeau P, Singh NR, Tarnus E and Bourdon E: The antioxidant properties of serum albumin. FEBS Lett 582: 1783-1787, 2008

39. Zoellner H, Siddiqui S, Kelly E and Medbury H: The anti-apoptotic activity of albumin for endothelium is inhibited by advanced glycation end products restricting intramolecular movement. Cell Mol Biol Lett 14: 575-586, 2009.

40. Bolitho C, Bayl P, Hou JY, et al: The anti-apoptotic activity of albumin for endothelium is mediated by a partially cryptic protein domain and reduced by inhibitors of $\mathrm{G}$-coupled protein and PI-3 kinase, but is independent of radical scavenging or bound lipid. J Vasc Res 44: 313-324, 2007.

41. Iwasaki T, Togashi Y and Terauchi Y: Significant association of serum albumin with severity of retinopathy and neuropathy, in addition to that of nephropathy, in Japanese type 2 diabetic patients. Endocr J 55: 311-316, 2008.

42. Autieri MV: Expression of anaphase-promoting complex 5 in balloon angioplasty-injured rat carotid arteries and mitogenstimulated human vascular smooth muscle cells. Biochem Biophys Res Commun 282: 723-728, 2001.

43. Ou H, Shen YH, Utama B, et al: Effect of nuclear actin on endothelial nitric oxide synthase expression. Arterioscler Thromb Vasc Biol 25: 2509-2514, 2005.

44. Funatsu H, Yamashita H, Noma H, Mimura T, Yamashita $T$ and Hori S: Increased levels of vascular endothelial growth factor and interleukin-6 in the aqueous humor of diabetics with macular edema. Am J Ophthalmol 133: 70-77, 2002.

45. Diz AP, Truebano $M$ and Skibinski DO: The consequences of sample pooling in proteomics: an empirical study. Electrophoresis 30: 2967-2975, 2009. 